\title{
Numerical Simulations of Solar Energetic Particle Event Timescales
}

\section{Associated with ICMES}

\author{
S.-Y. Qi ${ }^{1,2}$, G. Qin ${ }^{3,1,2}$ and Y. Wang ${ }^{3}$ \\ 1 State Key Laboratory of Space Weather, National Space Science Center, Chinese Academy of Sciences, \\ Beijing 100190, China \\ 2 College of Earth Sciences, University of Chinese Academy of Sciences, Beijing 100049, China \\ 3 School of Science, Harbin Institute of Technology, Shenzhen, 518055, China; qingang@hitsz.edu.cn
}

\begin{abstract}
Recently, S.W. Kahler studied the solar energetic particle (SEP) event timescales associated with coronal mass ejections (CMEs) from spacecraft data analysis. They obtained different timescales of SEP events, such as $T O$, the onset time from CME launch to SEP onset, $T R$, the rise time from onset to half the peak intensity $\left(0.5 I_{p}\right)$, and $T D$, the duration of the SEP intensity above $0.5 I_{p}$. In this work, we solve SEPs transport equation considering ICME shocks as energetic particle sources. With our modeling assumptions, our simulations show similar results to Kahler's spacecraft data analysis that the weighted average of $T D$ increases with both CME speed and width. Besides, from our simulation results, we suggest $T D$ is directly dependent on CME speed, but not dependent on CME width, which were not achieved from the observation data analysis.
\end{abstract}

Key words: Sun: particle emission — Sun: flare — Sun: coronal mass ejections (CMEs)

\section{INTRODUCTION}

Solar energetic particle (SEP) events could be mainly divided into two classes through duration and intensity. The short-duration and low-intensity events, which are called impulsive events, are considered to be produced by solar flares. On the other hand, the longer duration and higher intensity ones, which are called gradual events, are considered produced by coronal and interplanetary shocks driven by coronal mass ejections (CMEs). It is interesting to study the relationship between gradual SEP event properties and the characteristics of the associated CMEs. With the first-order Fermi acceleration mechanism (Zank et al., 2000) introduced an onion shell model using a one-dimensional hydrodynamic code for the evolution of the CME-driven shock in the Parker interplanetary magnetic field (IMF). The model is valid only in strong shocks due to Bohm diffusion coefficient used, so Rice et al. (2003) modified it to be usable in arbitrary strengths. In addition, Li et al. (2003) studied the transport of SEPs with their onion shell acceleration model considering particles pitch angle scattering without perpendicular diffusion. In their model, charged particles' pitch angle diffusion is not considered between two consecutive pitch angle scatterings. Furthermore, 
Verkhoglyadova et al. (2009, 2010) adopted this model to study individual SEP events caused by CME shocks, their simulation results can fit well with spacecraft observations for different elements. On the other hand, considering that the interplanetary coronal mass ejection (ICME) shocks can continuously accelerate SEPs when propagating outward, Kallenrode \& Wibberenz (1997); Kallenrode (2001) treated the ICME shock as a moving particle source. And the model was adopted in a numerical code 1 by Wang et al. (2012) to study ICME driven shock accelerated particles' transport in three dimensional solar wind and IMF including both parallel and perpendicular diffusion coefficients. Furthermore, under varying perpendicular diffusion and shock acceleration strength, Oin et al. (2013) reproduced the reservoir phenomenon with SPTC numerical simulations. In addition, with the same numerical modeling, Wang \& Qin (2015) researched the gradual SEP events spectra forcusing on the spatial and temporal invariance. Finally, Qin \& Wang (2015) compared the simulation results from SPTC with the multi-spacecraft (Helios 1, Helios 2, and IMP 8) observations during a gradual SEP event, and they obtained the SPTC simulations which best fit the SEP event observed by spacecraft located in different space.

To investigate the relationship between SEP event properties with the associated CMEs, Ding et al. (2014) studied the interaction of two CMEs erupted nearby during a large SEP event by multiple spacecraft observations with the graduated cylindrical shell model. And they obtained the solar particle release time and path length which indicated the necessary influence of the "twin-CME" (Li et al.,, 2012; Temmer et al., 2012) on the SEP event.

Because of the huge damage caused by SEPs, the study of peak intensities of SEPs becomes very important. Ding et al. (2015) presented the new observation results of peak intensity with Fe/O ratio, which indicate the role of seed population in extremely large SEPs. Reinard \& Andrews (2006) studied the dependence of the occurrence and peak intensities of SEP events with CME properties thoroughly using databases of the LASCO/SOHO CMEs and the GOES E $>10 \mathrm{MeV}$ protons. Besides peak intensities, timescales are another very important property of SEPs which could make contribution to both space weather forecasting and understanding of the SEP injection profiles and propagation characteristics.

In order to study the properties and associations of SEP events, Cane et al. (2010) compared SEPs with flares and CMEs of 280 solar proton events which extended above $25 \mathrm{MeV}$ occurred from 1997 to 2006 by near-Earth spacecraft. They divided the events into 5 groups according to the ratios $e / p$ and Fe/O at event onset. Their results suggested that SEP event occurrence and peak intensities are more likely to be associated with faster and wider CMEs, especially with western CME source regions. Furthermore, Pan et al. (2011) investigated SEP timescales, such as the SEP onset time, the SEP rise time, and the SEP duration. With an ice-cream cone model, Pan et al. (2011) studied LASCO/S OHO observation data of 95 CMEs associated with SEP events during 1998 - 2002, and came to conclusions that the SEP onset time has no significant correlation with the CME speed, nor with the CME width. They also suggested that the SEP rise time and the SEP duration have significantly positive correlations with the radial speed and angular width of the associated CMEs unless the events are not magnetically well connected to the Earth.

Kahler (2013) did a research on the relationship between the EPACT/Wind $20 \mathrm{MeV}$ SEP events timescales and their associated CME speed and widths observed by LASCO/SOHO. In Kahler (2013),

\footnotetext{
1 Hereafter, we denote the code as Shock Particle Transport Code, SPTC.
} 
217 SEP events observed in a solar cycle during the period 1996-2008 were used. They defined the three characteristic times of the SEP events. The time from inferred CME launch at $1 R_{\odot}$ to the time of the 20 MeV SEP onset at Wind was denoted as $T O$. The time from SEP onset to the time the intensity reached half of the peak value $\left(0.5 I_{p}\right)$ was denoted as $T R$. And the time during which the intensity was above $0.5 I_{p}$ was denoted as $T D$. From their results, they found that CME speed and width were of significant correlation and it is not easy to interpret the contribution of CME speed and width to timescales separately. Therefore, they suggested that faster and wider CMEs which drive shocks and accelerate SEPs over longer times would thus produce the longer SEP timescales $T R$ and $T D$.

In this paper, with the data used in the analysis of Kahler (2013), we study the CME timescales by numerical simulations with the SPTC, and we compare our results with that of Kahler (2013). In section 2 , we present the model. In section 3, we present the data analysis. In section 4, we show our results. In section 5, we present the conclusions and discussion.

\section{MODEL}

We model the transport of SEPs by following previous research (e.g., Qin et al., 2006; Zhang et al., 2009). The three-dimensional focused transport equation is written as (Skilling, 1971; Schlickeiser, 2002; Oin et al., 2006; Zhang et al., 2009)

$$
\begin{aligned}
& \frac{\partial f}{\partial t}= \nabla \cdot\left(\kappa_{\perp} \cdot \nabla f\right)+\frac{\partial}{\partial \mu}\left(D_{\mu \mu} \frac{\partial f}{\partial \mu}\right)-\left(v \mu \hat{\boldsymbol{b}}+\boldsymbol{V}^{s w}\right) \cdot \nabla f \\
&+p\left[\frac{1-\mu^{2}}{2}\left(\nabla \cdot \boldsymbol{V}^{s w}-\hat{\boldsymbol{b}} \hat{\boldsymbol{b}}: \nabla \boldsymbol{V}^{s w}\right)+\mu^{2} \hat{\boldsymbol{b}} \hat{\boldsymbol{b}}: \nabla \boldsymbol{V}^{s w}\right] \frac{\partial f}{\partial p} \\
& \quad-\frac{1-\mu^{2}}{2}\left[-\frac{v}{L}+\mu\left(\nabla \cdot \boldsymbol{V}^{s w}-3 \hat{\boldsymbol{b}} \hat{\boldsymbol{b}}: \nabla \boldsymbol{V}^{s w}\right)\right] \frac{\partial f}{\partial \mu},
\end{aligned}
$$

where $f(\boldsymbol{x}, \mu, p, t)$ is the gyrophase-averaged distribution function, $\boldsymbol{x}$ is the position in a non-rotating heliographic coordinate system, $\mu$ is the particle pitch-angle cosine, $p$ is the particle momentum, $v$ is the particle speed, $t$ is the time, $\kappa_{\perp}$ and $D_{\mu \mu}$ are the particle perpendicular and pitch-angle diffusion coefficients, respectively, $\boldsymbol{V}^{s w}=V^{s w} \hat{\boldsymbol{r}}$ is the solar wind velocity which is in the radial direction, and $L=\left(\hat{\boldsymbol{b}} \cdot \nabla \ln B_{0}\right)^{-1}$ is the magnetic focusing length determined by the magnitude of the background magnetic field $B_{0}$ and the unit vector along the local magnetic field $\hat{\boldsymbol{b}}$. In the equation (1), almost all important transport effects are included, i.e., perpendicular diffusion (1st term in RHS), pitch angle diffusion (2nd term in RHS), particle streaming along field line and solar wind flowing in the IMF (third term in RHS), adiabatic cooling in the expanding solar wind (4th term in RHS), and magnetic focusing in the diverging IMF (5th term in RHS). Here, the drift effects are neglected for lower-energy SEP transport in the inner heliosphere. Also the IMF is modeled with the Parker field.

By following Burger et al. (2008), diffusion coefficients are determined. We set the perpendicular diffusion coefficient from the nonlinear guiding center (NLGC) theory (Matthaeus et al., 2003) approximated with the analytical form according to Shalchi et al. (2004, 2010),

$$
\boldsymbol{\kappa}_{\perp}=v l_{d}^{2 / 3} \lambda_{\|}^{1 / 3}(\mathbf{I}-\hat{\mathbf{b}} \mathbf{b})
$$

where $l_{d}$ is a parameter to control the value of the perpendicular diffusion coefficient. For simplicity, $\boldsymbol{\kappa}_{\perp}$ is set to be independent of $\mu$ with the assumption that particle pitch-angle diffusion is much faster than 
perpendicular diffusion, but generally $\mu$ dependent perpendicular diffusion coefficient should be used (e.g., Qin \& Shalchi, 2014b).

The parallel particle mean free path (mfp) $\lambda_{\|}$is written as (Jokipii, 1966; Hasselmann \& Wibberenz, 1968; Earl, 1974)

$$
\lambda_{\|}=\frac{3 v}{8} \int_{-1}^{+1} \frac{\left(1-\mu^{2}\right)^{2}}{D_{\mu \mu}} d \mu,
$$

and parallel diffusion coefficient $\boldsymbol{\kappa}_{\|}$can be written as $\boldsymbol{K}_{\|}=v \lambda_{\|} / 3$.

We follow Beeck \& Wibberenz (1986) and Teufel \& Schlickeiser (2003) to model the pitch angle diffusion coefficient

$$
D_{\mu \mu}(\mu)=G v R_{L}^{s-2}\left\{|\mu|^{s-1}+h\right\}\left(1-\mu^{2}\right),
$$

where $G$ is a parameter to control the value of $D_{\mu \mu}(\mu), v$ is the particle speed, $R_{L}=p c /\left(|q| B_{0}\right)$ is the particle Larmor radius. Here, a larger value of $h=0.01$ is chosen for non-linear effect of pitch angle diffusion at $\mu=0$ in the solar wind (Qin \& Shalchi, 2009, 2014a).

To model the particle injection, the shock is treated as a moving SEP source with the boundary condition Kallenrode \& Wibberenz, 1997):

$$
f_{b}=a \delta\left(r-v_{s} t\right)\left(\frac{r}{r_{c}}\right)^{\alpha} \exp \left[-\frac{|\phi(\theta, \varphi)|}{\phi_{c}(p)}\right] p^{-\gamma} H\left(\phi_{s}-|\phi(\theta, \varphi)|\right)
$$

where $\alpha$ and $\phi_{c}$ are the shock acceleration strength parameters. We assume $\phi_{c}$ as a constant, but $\alpha$ as a function of shock speed, e.g., we set

$$
\alpha= \begin{cases}-3.5 & \text { if } v_{\mathrm{s}}<v_{1} \\ v_{s} / v_{0}-5 & \text { if } v_{1} \leq v_{\mathrm{s}} \leq v_{2} \\ -2 & \text { if } v_{\mathrm{s}}>v_{2}\end{cases}
$$

where $v_{0}=500 \mathrm{~km} \mathrm{~s}^{-1}, v_{1}=750 \mathrm{~km} \mathrm{~s}^{-1}$, and $v_{2}=1500 \mathrm{~km} \mathrm{~s}^{-1} . \phi(\theta, \varphi)$ is the angle between source center and any point of particle injection $(\theta, \varphi) \cdot \gamma$ is the spectral index of source particles. In the simulations, we inject energetic particle shells with small space intervals $\Delta r . H(x)$ is the Heaviside step function, with $\phi_{s}$ being the half angular width of the shock. A more detailed description of the shock model of our simulations can be referred to Wang et al. (2012).

The transport equation (1) is solved by a time-backward Markov stochastic process method (Zhang, 1999) in the simulations. And the detailed description of the method can be referred to Oin et al. (2006). As mentioned in section 1 our numerical code of transport of energetic particles with the CME driven shock as a moving particle source is denoted as Shock Particle Transport Code, i.e., SPTC.

\section{DATA ANALYSIS}

We investigate $20 \mathrm{MeV}$ proton intensity-time profiles of SEP events during 1996 to 2008 with their associated CMEs. In particular, the SEP data is from EPACT (the Energetic Particles: Acceleration, Composition, and Transport) (von Rosenvinge et al., 1995) experiment on the Wind spacecraft, and the information of their related CMEs is observed by $\mathrm{SOHO}$ (the Solar and Heliospheric Observatory mission) LASCO (Large Angle and Spectrometric Coronagraph) (Brueckner et al., 1995). Of the total 217 SEPs during this period (Kahler, 2013), we study 204 SEPs whose CME parameters are available. In addition, for each event, the CME solar source is determined by flare location, and the speed $\left(v_{C M E}\right)$ and width $\left(W_{C M E}\right)$ of CME are obtained from Kahler (2013). 


\subsection{Parameter Selection}

For the grouping and selecting data, we follow the method suggested by Kahler (2013) dividing the 204 events into five longitude ranges with about 41 events each, and subdividing each longitude range into several groups sorted on $v_{C M E}$ and $W_{C M E}$, respectively. The median values of longitude, $v_{C M E}$, and $W_{\text {shock }}$ in each group are used as the characteristic values.

From data analysis of spacecraft observations, it is not easy to identify SEP onset time accurately which is usually covered by the background of intensity. Therefore, in this work, we only focus on the variation of TD with $v_{C M E}$ and $W_{C M E}$. To compare with the observation, we obtain the data analysis results of variation of TD with $v_{C M E}$ and $W_{C M E}$ from Kahler (2013) as shown in Table1 From Table 1 we can see, we study SEP events with source location longitude in three ranges, W33-W60, W62-W90, and W100-bWL, with median values W48, W77, and W112, respectively. Note that bWL indicates sources behind the west limbs. In each range of longitude, $T D$ is shown as varying with the median values of $v_{C M E}$ and $W_{C M E}$ by subdividing the range into several groups sorted on $v_{C M E}$ and $W_{C M E}$, respectively.

Table 1 The data analysis results of variation of TD with $v_{C M E}$ and $W_{C M E}$ from Kahler (2013).

\begin{tabular}{|c|c|c|c|c|c|c|}
\hline Source Location & \multicolumn{2}{|c|}{ W33-W60 } & \multicolumn{2}{|c|}{ W62-W90 } & \multicolumn{2}{|c|}{ W100-bWL } \\
\hline \multirow{5}{*}{$\begin{array}{l}\text { TD varying } \\
\text { with } v_{C M E}\end{array}$} & $v_{C M E}(\mathrm{~km} / \mathrm{s})$ & $\mathrm{TD}(\mathrm{h})$ & $v_{C M E}(\mathrm{~km} / \mathrm{s})$ & $\mathrm{TD}(\mathrm{h})$ & $v_{C M E}(\mathrm{~km} / \mathrm{s})$ & $\mathrm{TD}(\mathrm{h})$ \\
\hline & 450 & 6.3 & 650 & 6.5 & 620 & 13.2 \\
\hline & 800 & 12.0 & 1150 & 9.8 & 900 & 14.0 \\
\hline & 1175 & 8.8 & 1450 & 21.3 & 1325 & 12.5 \\
\hline & 1600 & 14.5 & 2100 & 18.1 & 1750 & 17.0 \\
\hline \multirow{4}{*}{$\begin{array}{l}\text { TD Varying } \\
\text { with } W_{C M E}\end{array}$} & $W_{C M E}\left(^{\circ}\right)$ & $\mathrm{TD}(\mathrm{h})$ & $W_{C M E}\left(^{\circ}\right)$ & $\mathrm{TD}(\mathrm{h})$ & $W_{C M E}\left({ }^{\circ}\right)$ & $\mathrm{TD}(\mathrm{h})$ \\
\hline & 77 & 8.3 & 133 & 7.8 & 100 & 7.5 \\
\hline & 208 & 11.3 & 171 & 15.0 & 178 & 13.3 \\
\hline & 360 & 15.8 & 360 & 16.4 & 360 & 17.2 \\
\hline
\end{tabular}

In order to study SEP timescales associated with CMEs, we use the SPTC described in Section 2 to simulate the transport of SEPs assuming the CME shock as a moving particle source and that the shock nose is in the flare direction relative to the solar center. In SPTC, the speed of shock, $v_{s}$, and the width of shock, $W_{s}$, are needed. While, in the spacecraft data analysis of Kahler (2013) the speed and width of CME are used instead. To compare the simulation results with the spacecraft data analysis, we need a model for relationship between $v_{s}$ and $v_{C M E}$, and that for relationship between $W_{s}$ and $W_{C M E}$. Firstly, we assume the speed of CME is the same as that of shock, $v_{s}=v_{C M E}$. Secondly, since the width of shock $\left(W_{s}\right)$ is larger than that of CME $\left(W_{C M E}\right)$,we set,

$$
W_{s}= \begin{cases}W_{C M E}+\Delta W & \text { if } W_{\mathrm{CME}}<360^{\circ}-\Delta \mathrm{W} \\ 360^{\circ} & \text { otherwise. }\end{cases}
$$

By testing several value of $\Delta W$, we finally set $\Delta W=90^{\circ}$. It is noted that such kind of model for $W_{s}$ is only an approximation, and it could lead to the discrepancy between the observation and simulation results. So we need to use a better $W_{s}$ model in the future. Generally, the event source is near the solar equator, so the characteristic latitude of source location is set as $10^{\circ}$ north. Other important simulation parameters not varying are shown in Table 2 . 
Table 2 Model Parameters Used in the Calculations.

\begin{tabular}{|l|l|l|}
\hline Parameter & Physical meaning & Value \\
\hline \hline$E$ & Particles energy & $20 \mathrm{MeV}$ \\
\hline$r_{O}$ & Observer solar distance & $1 \mathrm{AU}$ \\
\hline$\Delta r$ & Shock space interval between two fresh injections & $0.001 \mathrm{AU}$ \\
\hline$r_{c}$ & Radial normalization parameter & $0.05 \mathrm{AU}$ \\
\hline$\gamma$ & Spectral index of source particles & -3.5 \\
\hline$\phi_{c}$ & Shock strength parameter & $15^{\circ}$ \\
\hline$\lambda_{\|}^{\mathrm{a}}$ & Particle mean free path & $0.16 \mathrm{AU}$ \\
\hline$\kappa_{\perp} / \kappa_{\|}^{\mathrm{a}}$ & Ratio between perpendicular and parallel diffusion coefficient & $6.1 \%$ \\
\hline$r_{b 0}$ & Inner boundary & $0.05 \mathrm{AU}$ \\
\hline$r_{b 1}$ & Outer boundary & $50 \mathrm{AU}$ \\
\hline
\end{tabular}

a For $20 \mathrm{MeV}$ protons in the ecliptic at $1 \mathrm{AU}$.

In order to investigate the relationship between solar wind speed $v_{S W}$ and CME speed $v_{C M E}$, we obtain $v_{S W}$ observation data from Wind spacecraft for the $204 \mathrm{CME}$ events to fit the relationship between $v_{C M E}$ and $v_{S W}$. It is shown that $v_{C M E}$ and $v_{S W}$ are positively correlated. As we assumed above that $v_{S}=v_{C M E}$, the relationship between $v_{C M E}$ and $v_{S W}$ would turn to that between $v_{S}$ and $v_{S W}$. Thus $v_{S W}$ can be represented by $v_{s}$ as

$$
v_{S W}=1.77 \times 10^{-5} v_{s}^{2}+425,
$$

here, $v_{S W}$ and $v_{s}$ are in the unit of $\mathrm{km} \mathrm{s}^{-1}$. We also divide the events into several groups sorted on $v_{C M E}$, and obtain the median values of $v_{C M E}$ as the characteristic ones for each group. So we obtain the counterpart values $v_{s}$ and $v_{S W}$ through the assumption above. And we use the characteristic ones in the simulations shown in Table 3 The Table 3 also shows the other input parameters in each simulation coming from the characteristic values of $v_{C M E}, W_{C M E}$ and source location longitude picked up from Kahler (2013) shown in Table 1.

\subsection{Simulation Output}

For each data point, 3200000 virtual particles are calculated in our simulations. In our simulations, we obtain the time profiles of SEPs with characteristic speed and width of CME, with which we can get the SEP timescale, TD. For example, in Figure1, we show simulation results of $20 \mathrm{MeV}$ proton flux during an SEP event. In the simulation of Figure 1 we set solar wind speed as $502.2 \mathrm{~km} \mathrm{~s}^{-1}$, longitude as 48 degrees west, CME speed as $1600 \mathrm{~km} \mathrm{~s}^{-1}, \mathrm{CME}$ width as $180^{\circ}$, other parameters are shown in Table 2. In Figure1, the dotted line indicates the peak intensity $\left(I_{p}\right)$ of the event, and the dash-dotted line indicates the half peak intensity. $T_{s}$ and $T_{e}$ indicate the earliest and latest time when the intensity is half peak, respectively. So we can obtain $T D=T_{e}-T_{s}$ from the time profile of intensity of simulation results.

From the results of the simulations we can also get the weighted averages as following. For example, in each range of shock speed and longitude, we have three ranges of shock width, so we have three values 
Table 3 Shock speed and width, and solar wind speed used in all simulations.

\begin{tabular}{|c|c|c|c|c|c|c|c|c|}
\hline \multicolumn{9}{|c|}{ Source location } \\
\hline \multicolumn{3}{|c|}{ N10W48 } & \multicolumn{3}{|c|}{ N10W77 } & \multicolumn{3}{|c|}{ N10W115 } \\
\hline$v_{s}(\mathrm{~km} / \mathrm{s})$ & $v_{S W}(\mathrm{~km} / \mathrm{s})$ & $W_{s}\left({ }^{\circ}\right)$ & $v_{s}(\mathrm{~km} / \mathrm{s})$ & $v_{S W}(\mathrm{~km} / \mathrm{s})$ & $W_{s}\left({ }^{\circ}\right)$ & $v_{s}(\mathrm{~km} / \mathrm{s})$ & $v_{S W}(\mathrm{~km} / \mathrm{s})$ & $W_{s}\left(^{\circ}\right)$ \\
\hline 450 & 433.5 & 167 & 650 & 433.5 & 223 & 620 & 433.5 & 190 \\
\hline 450 & 433.5 & 298 & 650 & 433.5 & 261 & 620 & 433.5 & 268 \\
\hline 450 & 433.5 & 360 & 650 & 433.5 & 360 & 620 & 433.5 & 360 \\
\hline 800 & 455.4 & 167 & 1150 & 455.4 & 223 & 900 & 455.4 & 190 \\
\hline 800 & 455.4 & 298 & 1150 & 455.4 & 261 & 900 & 455.4 & 268 \\
\hline 800 & 455.4 & 360 & 1150 & 455.4 & 360 & 900 & 455.4 & 360 \\
\hline 1175 & 444.1 & 167 & 1450 & 444.1 & 223 & 1325 & 444.1 & 190 \\
\hline 1175 & 444.1 & 298 & 1450 & 444.1 & 261 & 1325 & 444.1 & 268 \\
\hline 1175 & 444.1 & 360 & 1450 & 444.1 & 360 & 1325 & 444.1 & 360 \\
\hline 1600 & 502.2 & 167 & 2100 & 502.2 & 223 & 1750 & 502.2 & 190 \\
\hline 1600 & 502.2 & 298 & 2100 & 502.2 & 261 & 1750 & 502.2 & 268 \\
\hline 1600 & 502.2 & 360 & 2100 & 502.2 & 360 & 1750 & 502.2 & 360 \\
\hline
\end{tabular}

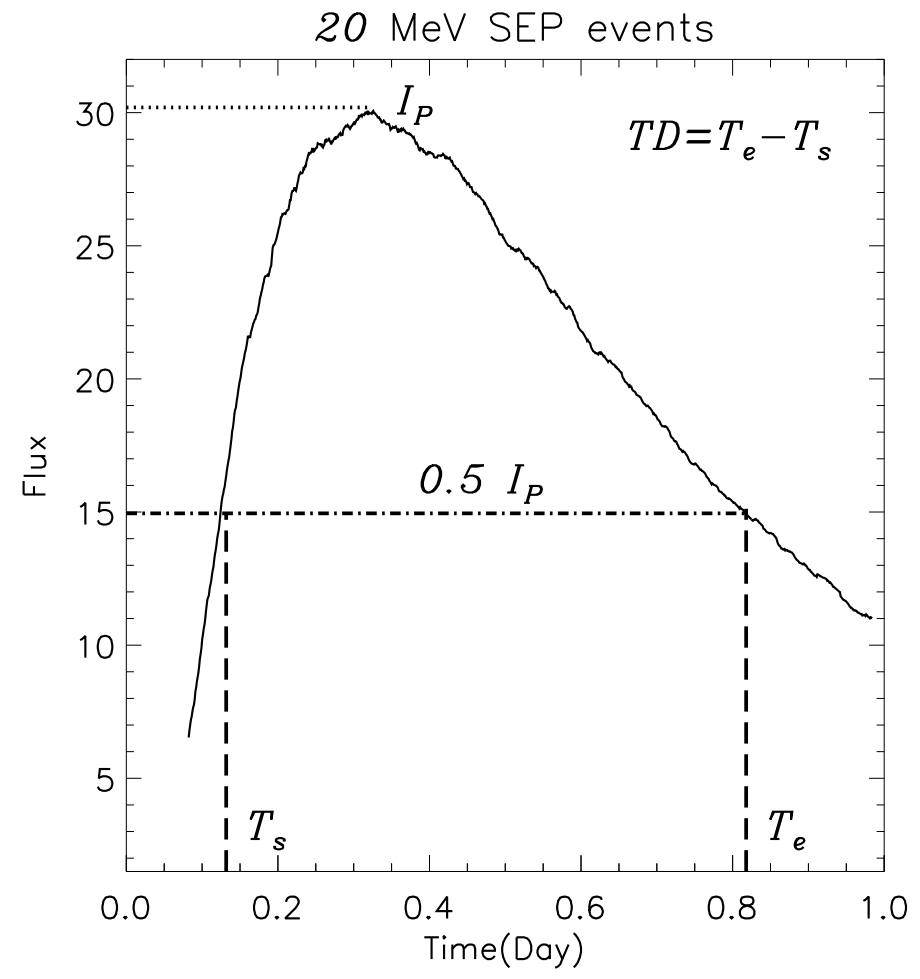

Fig. 1 Flux of $20 \mathrm{MeV}$ proton during an SEP event with parameters shown in the text. The dotted line indicates the peak intensity of the event, and the dash-dotted line indicates the half peak intensity. $T_{s}$ and $T_{e}$ indicate the earliest and latest time when the intensity is half peak, respectively. 
three ranges of shock width we can get their percentage according to the number of events, with which the weighted value of $T D$ is obtained from the individual values of $T D$.

Further, we study the relationship between CME speed and CME width using the observation data in Kahler (2013). We subdivide each longitude range into several groups sorted on CME width. We get average CME speed for each group. The results are shown in Figure 2 as the relationship between average of CME speed and the median value of CME width. The three data points in each longitude group of Figure 2 match those of the three CME width bins of Table 1. The line indicates fitting of the data. It is found that in statistics the average CME width increases with the increasing of CME speed.

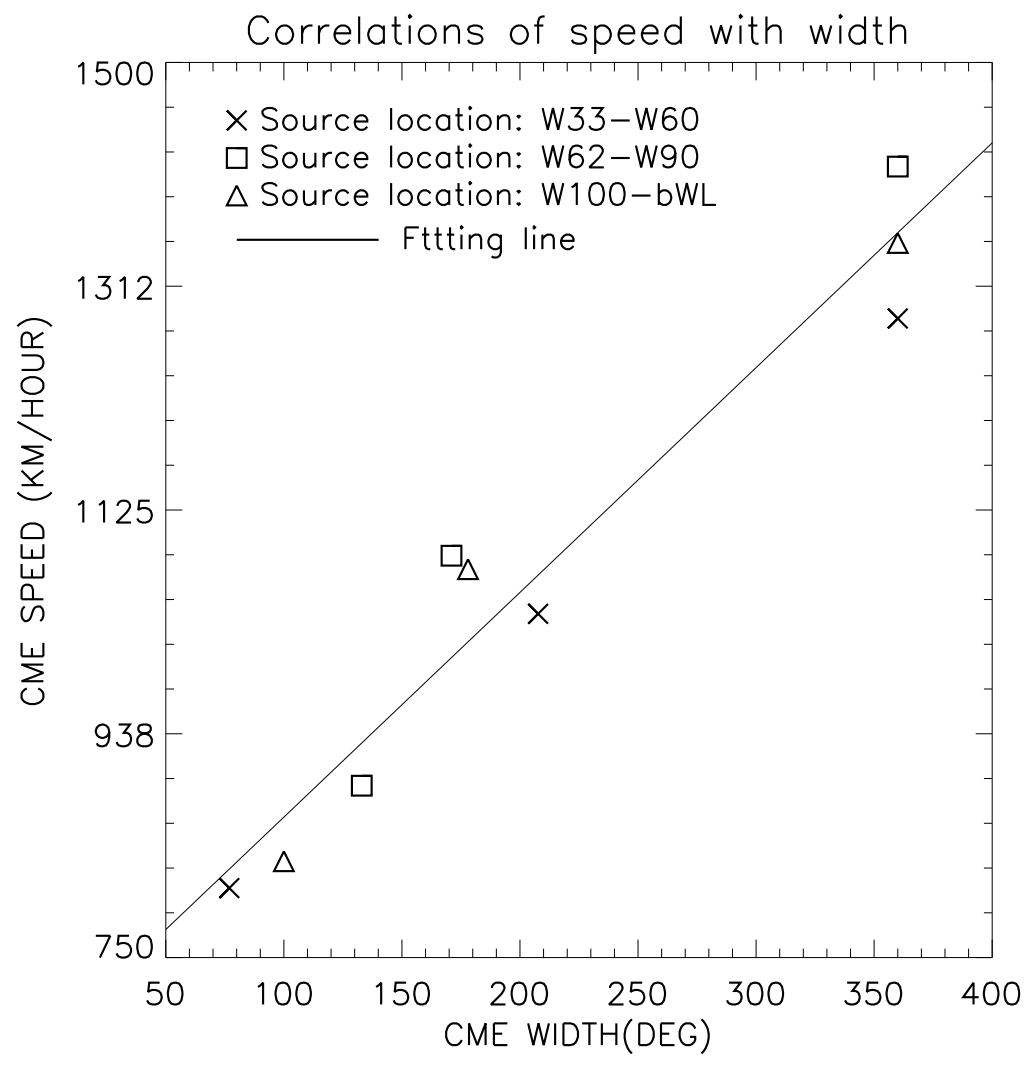

Fig. 2 Variations of CME speed as a function of CME width with different source location. The crosses indecate $\mathrm{CME}$ speed averages of the CME width ranges in the source location range of W33-W60, and the squares are that of W62-W90, the triangles are that of W100-bWL. The line indicates fitting of the data. The symbols are from observation data analysed by Kahler (2013).

\section{RESULTS}

Figure 3 shows SEP timescale $T D$ vs. CME speed for $20 \mathrm{MeV}$ SEP events detected at 1 AU with different source locations in different pannels. The top, middle and bottom panels show different longitudes of source locations, $48^{\circ}$ west, $77^{\circ}$ west, and $115^{\circ}$ west, respectively. The black squares indicate spacecraft observation data in Table 1 which are obtained from the data analysis of Kahler (2013). The TD and CME speed for each data points correspond to those of Table 1 The red triangles indicate the weighted average of simulations according to the distribution of number of events with different CME widths for any given CME speed 
interval obtained from the observation data in Kahler (2013) corresponding to the the abscissa of black squares. The red and black dashed lines indicate the linear fitting of the weighted average simulation results represented by the red triangles and that of the spacecraft observation data represented by the black squares, respectively. From Figure 3 we can see, the simulation results show the similar trend of observation data, that is, the SEP timescale TD increases with CME speed.

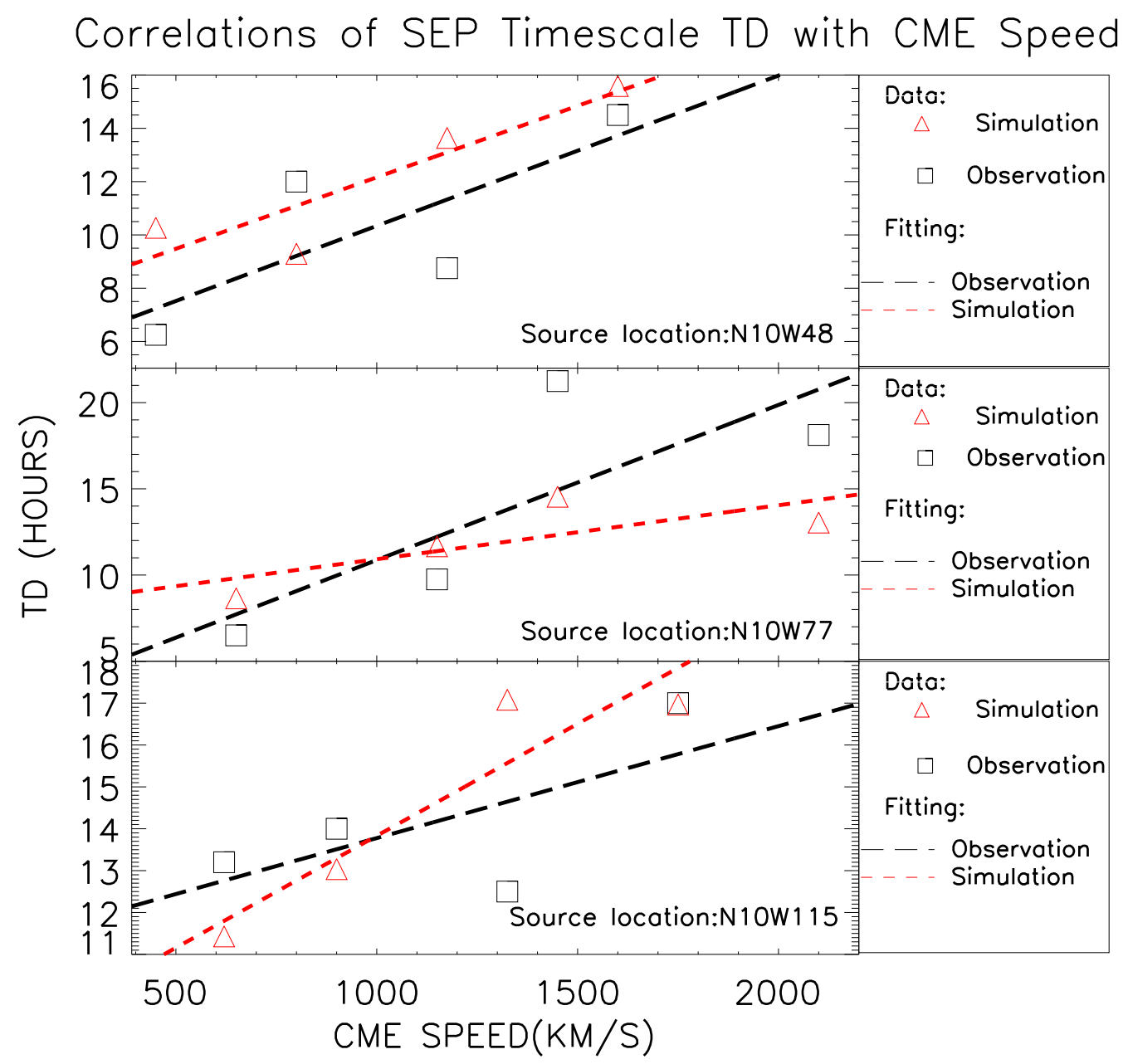

Fig. 3 SEP event timescale $T D$ vs. CME speed. Different panels indicate different source location. The black squares are from observation data analysed by Kahler (2013). The $T D$ and CME speed for each data points correspond to those of Table 1, The red triangles indicate weighted average of simulation results. The black dashed lines indicate linear fitting of observation data. The red dashed lines indicate linear fitting of the weighted average of simulation results.

Figure 4 shows plot similar as Figure 3 except that $\mathrm{x}$-coordinate is CME width. The value for each black squares correspond to those of Table 1 The red triangles indicate weighted average of simulation results according to the distribution of number of events with different CME speeds for any given CME width interval obtained from the observation data in Kahler (2013) corresponding to the abscissa of black squares. Similarly as in Figure 3 the red and black dashed lines indicate the linear fitting of the weighted average 
of simulation results and that of the spacecraft observation data, respectively. From Figure 4 we can see, generally, the simulation results show the similar trend of observation data but with less slope, that is, the SEP timescale TD increases with CME width. However, from top panel of Figure 4(N10W48) it is shown that, the observation results shows the SEP timescale TD increases with CME width, but the simulation results shows constant for different CME width. It is noted that our simulations could show deviation from observations due to modeling and statistical problems.

\section{Correlations of SEP Timescale TD with CME Width}

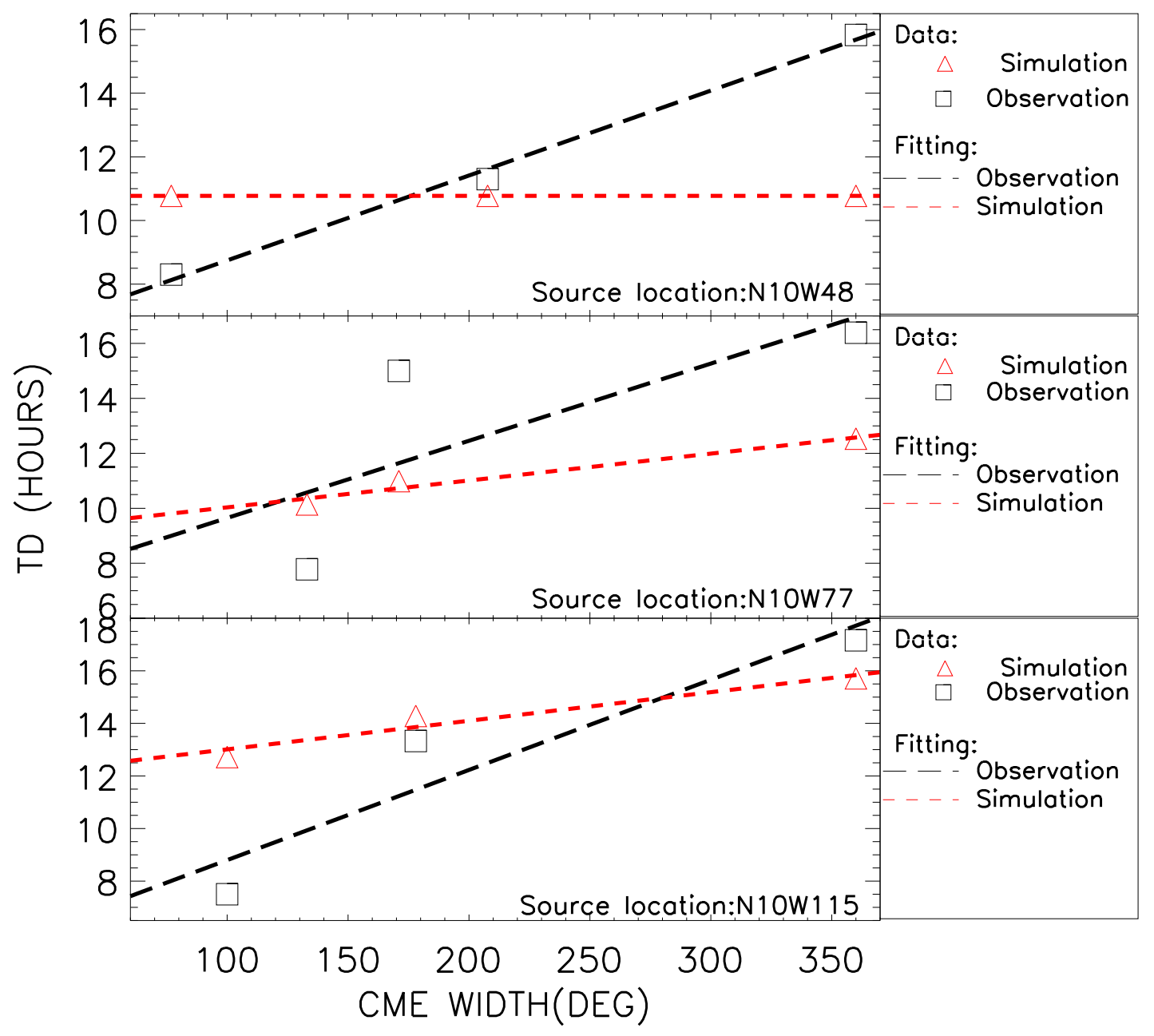

Fig. 4 SEP event timescale $T D$ vs. CME width. Different panels indicate different source location. The black squares are from observation data analysed by Kahler (2013). The TD and CME width for each data points correspond to those of Table 1. The red triangles indicate weighted average of simulation results. The black dashed lines indicate linear fitting of observation data. The red dashed lines indicate linear fitting of the weighted average of simulation results.

For further study on the contribution of CME speed and width to timescales separately, we plot the individual simulations and weighted average of simulation results as follows. 
Figure 5 shows simulations of SEP timescale $T D$ vs. CME speed for $20 \mathrm{MeV}$ SEP events detected at $1 \mathrm{AU}$ with different source locations in different pannels. Similar as Figure 3 , the top, middle and bottom panels show different longitudes of source locations, $48^{\circ}$ west, $77^{\circ}$ west, and $115^{\circ}$ west, respectively. The yellow, green, and blue triangles indicate simulations with different CME widths corresponding to those of Table 1. The each data point of the yellow line shows a individual simulation with a distinct CME speed but a common CME width $133^{\circ}$ and source location of N10W48, and so are the green and bule lines with other source location. Besides, the value of all data points are shown in Table 3 The red triangles indicate the weighted average of simulations according to the distribution of number of events with different CME widths for any given CME speed interval obtained from the observation data in Kahler (2013).

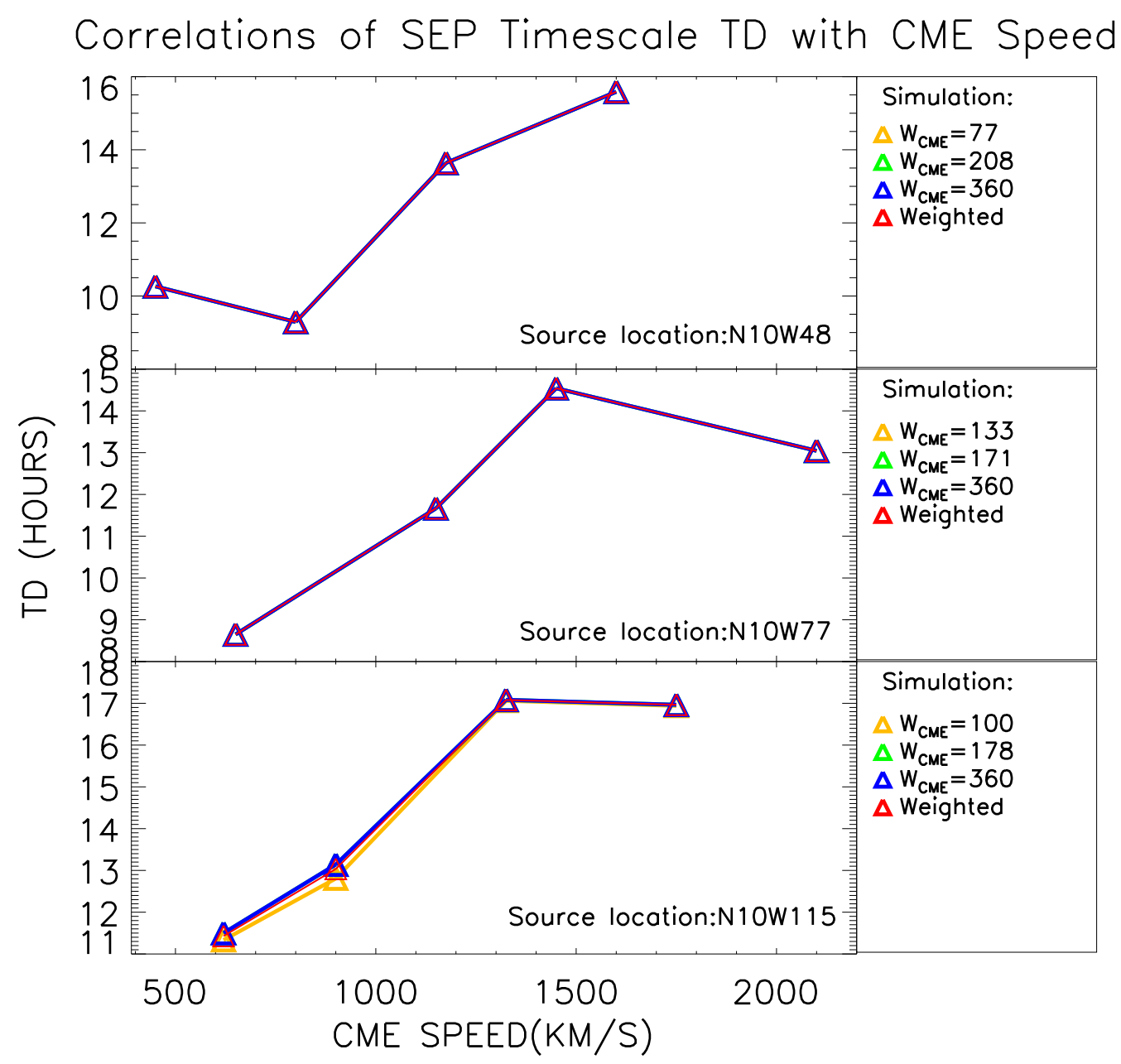

Fig. 5 Simulations of SEP event timescale $T D$ vs. CME speed. Different panels indicate different source location. The yellow, green and blue triangles indicate simulations with different CME widths. The red triangles indicate weighted average of simulation results.

From simulations of Figure 5 we can see, every single colored line increases, that is to say, for the same CME width, $T D$ generally increases with the increasing of CME speed, and the weighted average of $T D$ from simulations also generally increases with the increasing of CME speed. From the other aspect, the 
colored lines and symbols are almost overlap, from triangles with a common abscissa but different colors we can see, when CME speed is fixed, $T D$ with different CME widths are almost same, meanwhile, when CME width is fixed, $T D$ with different CME speeds are increased. So we suggest from our simulation that $T D$ is dependent on CME speed but not on CME width, which analysis of Kahler (2013) could not pick out.

Figure 6 shows plot similar as Figure 5except that $\mathrm{x}$-coordinate is CME width. The yellow, green, light blue and purple triangles indicate simulations with different CME speeds corresponding to those of Table 1 The each data point of the yellow line shows a individual simulation with a distinct CME width but a common CME speed $450 \mathrm{~km} \mathrm{~s}^{-1}$ and source location of N10W48, and so are the green and bule lines with other source location. Besides, the value of all data points are shown in Table 3 The red triangles indicate the weighted average of simulations according to the distribution of number of events with different CME speeds for any given CME width interval obtained from the observation data in Kahler (2013).

\section{Correlations of SEP Timescale TD with CME Width}

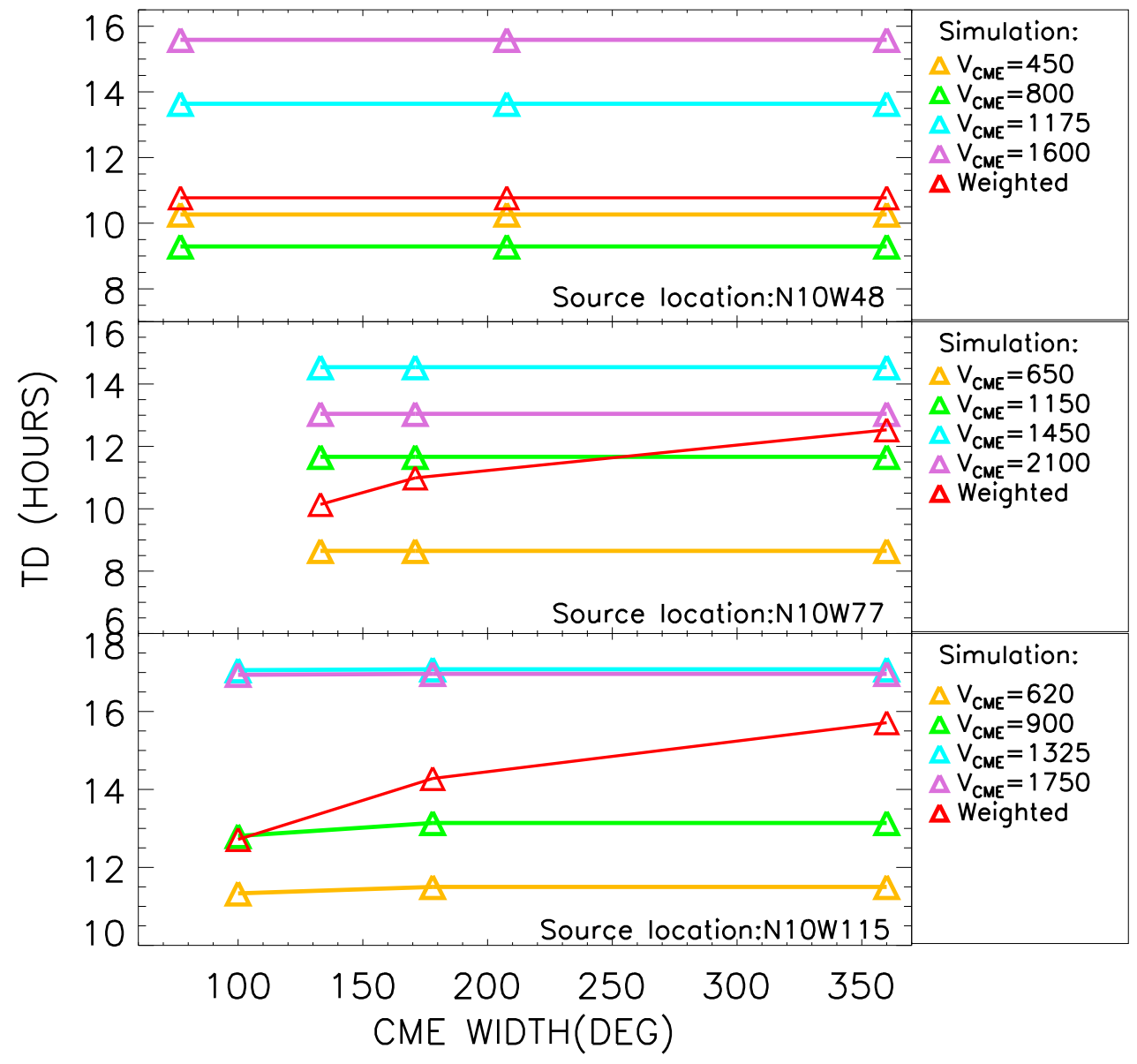

Fig. 6 Simulations of SEP event timescale $T D$ vs. CME width. Different panels indicate different source location. The yellow, green, light blue, and purple triangles indicate simulations with different CME speed. The red triangles indicate weighted average of simulation results. 
From simulations of Figure 6 we can see, the yellow, green, light blue and purple lines are almost aclinic, that is to say, for the same CME speed, $T D$ generally keeps constant with different CME width. However, the red line which combines each individual line connecting data points of common CME speed simulations with weighted average increases, that is to say, for the same CME width, $T D$ increases with the increasing of CME speed. In addition, the weighted average of $T D$ increases with the increasing of CME width. The reason is that with larger CME width it is more likely that CME speed becomes larger, so the weighted average of $T D$ becomes larger consequently.

\section{CONCLUSIONS AND DISCUSSION}

Generally, the accurate measurement of the first arriving particles in SEP events depends on the level of SEP flux background, so usually it is difficult to determine the timescales $T R, T O$, and $T O+T R$. However, $T D$, which indicates the duration of the SEP intensity above $0.5 I_{p}$, has nothing to do with the first arriving particles, so the measurements of $T D$ are relatively accurate. Therefore, we only study the timescale $T D$, but do not study $T R, T O$, or $T O+T R$.

In this work, we use the SPTC to simulate the transport of SEPs assuming the ICME shock as a moving particle source with parameters obtained from spacecraft observations analysed by Kahler (2013), and other parameters set as typical values of SEP events. From simulations we get SEP timescale $T D$ and compare with $T D$ values from spacecraft data analysis by Kahler (2013). From spacecraft observations shown in Kahler (2013) we obtain the contribution of CME speed with the same CME width, and we also obtain that of CME width with the same CME speed. Finally, from simulation results of $T D$ we can obtain the average of $T D$ weighted with the observations contribution.

Our simulations show that with the same CME speed, $T D$ keeps constant with the increasing of CME width, but that the weighted average of $T D$ increases with the increasing of CME width. From spacecraft data analysis in Kahler (2013) it is shown that $T D$, which is actually weighted average, increases with the increasing of CME width. In addition, our simulations show that with the same CME width, $T D$ increases with the increasing of CME speed, and that the average of $T D$ increases with the increasing of CME width. It is also shown in Kahler (2013) with spacecraft data analysis that the weighted average of $T D$ increases with the increasing of CME speed. Our simulations generally agree with spacecraft observations data analysis of Kahler (2013) that the weighted average of $T D$ increase with both CME speed and width. Furthermore, with our modeling assumptions, our simulations show some results not shown in Kahler (2013) that $T D$ is dependent directly on CME speed, but independent on CME width.

In order to study whether TD increases with CME width or speed by using observation data, one should choose SEP events with same CME speed but different CME width to show if TD increases with CME width, and also one should choose SEP events with same CME width but different CME speed to show if TD increases with CME speed. Kahler (2013) did not do it because of limitation of events number. But simulations do not have this limitation, and that offers us physical insights behind the observations. We compare the weighted average of simulation to the result of Kahler (2013), and we can show the trend of our weighted average generally agrees with the result of Kahler (2013), so our work do not contradict the 
observation result of Kahler (2013). Meanwhile, our individual results can be used to show if TD depends on CME width with same CME speed.

The model we use to calculate flux includes many effects, such as the source, parallel and perpendicular diffusion, adiabatic cooling, etc., the overall effects could be very complicated, so we have to use numerical simulations to get the results. It is possible in some cases TD would decrease. But generally, TD has a trend to increase with the same CME width and increasing CME speed, and TD has a trend to be constant with the same CME speed and increasing CME width. Here, we compare the general trend between observations and simulations.

We choose shock model conditions to favor larger particle injections with increasing speeds and widths in order to compare with observations. There are some parameters arbitrarily chosen and fixed in all simulations, we tried different parameters, for example, we tested simulations with different value of shock strength parameter $\phi_{c}$, such as $10^{\circ}, 15^{\circ}, 18^{\circ}$, and $25^{\circ}$, and we found they would not change our general results. In the future, we would continue to study the parameter effects in our model.

The observational evidences of the first detected SEP onsets or releases associated with the good magnetic connection to source were discussed in Ding et al. (2016). Besides, Rouillard et al. (2011, 2012) suggested that SEP onsets could be considered associated with the modeled first connections of field lines to shocks. On the other hand, Qin \& Wang (2015) showed the onsets from SPTC simulation results can fit well with that from observations of HELIOS 1, HELIOS 2, and IMP 8 at different longitudes simultaneously with perpendicular diffusion. It is interesting to compare the effects of these models carefully in the future.

There are many authors working on numerical simulations to produce SEP profiles from the shock onion shell model (e.g., Verkhoglyadova et al., 2009, 2010; Wang et al., 2012; Qin et al., 2013), and they usually study the individual SEPs in detail, in this work, however, we are trying to study many SEPs with simulations so we can compare with observations statistically. CME width data from Kahler (2013) were observed by only one satellite, $S O H O$, so they are lack of determinacy. In the future, we would study the CME data of multi-spacecraft observations. In addition, we would study peak intensity of gradual SEP events associated with CMEs by comparing the simulations of SPTC with the spacecraft data analysis (e.g., Kahler \& Vourlidas, 2013).

Acknowledgements We are partly supported by grants NNSFC 41304135, NNSFC 41574172, NNSFC 41374177, and NNSFC 41125016, the CMA grant GYHY201106011, and the Specialized Research Fund for State Key Laboratories of China. The computations were performed by Numerical Forecast Modeling R\&D and VR System of State Key Laboratory of Space Weather and Special HPC work stand of Chinese Meridian Project. CME data were taken from the CDAW LASCO catalog, which is generated and maintained at the CDAW Data Center by NASA and The Catholic University of America in cooperation with the Naval Research Laboratory. $S O H O$ is a project of international cooperation between ESA and NASA. We thank D. Reames for the use of the EPACT proton data.

\section{References}

Beeck, J., \& Wibberenz, G. 1986, Astrophysical Journal, 311, 437

Brueckner, G. E., Howard, R. A., Koomen, M. J., et al. 1995, Solar Physics, 162, 357 
Burger, R. A., Krüger, T. P. J., Hitge, M., \& Engelbrecht, N. E. 2008, Astrophysical Journal, 674, 511

Cane, H. V., Richardson, I. G., \& Von Rosenvinge, T. T. 2010, Journal of Geophysical Research, 115, A08101

Ding, L.-G., Cao, X.-X., Wang, Z.-W., \& Le, G.-M. 2016, Research in Astronomy and Astrophysics, 16, 122

Ding, L.-G., Li, G., Le, G.-M., Gu, B., \& Cao, X.-X. 2015, The Astrophysical Journal, 812, 171

Ding, L.-G., Li, G., Jiang, Y., et al. 2014, The Astrophysical Journal, 793, L35

Earl, J. A. 1974, The Astrophysical Journal, 193, 231

Hasselmann, K., \& Wibberenz, G. 1968, Z. Geophys., 34, 353

Jokipii, J. R. 1966, The Astrophysical Journal, 146, 1

Kahler, S. W. 2013, The Astrophysical Journal, 769, 110

Kahler, S. W., \& Vourlidas, A. 2013, The Astrophysical Journal, 769, 143

Kallenrode, M. B. 2001, Journal of Geophysical Research, 106, 24989

Kallenrode, M. B., \& Wibberenz, G. 1997, Journal of Geophysical Research, 102, 22311

Li, G., Moore, R., Mewaldt, R. A., Zhao, L., \& Labrador, A. W. 2012, Space Sci. Rev., 171, 141

Li, G., Zank, G. P., \& Rice, W. K. M. 2003, Journal of Geophysical Research, 108, 1082

Matthaeus, W. H., Qin, G., Bieber, J. W., \& Zank, G. P. 2003, Astrophysical Journal, 590, L53

Pan, Z., Wang, C., Wang, Y., \& Xue, X. 2011, Solar Physics, 270, 593

Qin, G., \& Shalchi, A. 2009, Astrophysical Journal, 707, 61

Qin, G., \& Shalchi, A. 2014a, Physics of Plasmas, 21, 231

Qin, G., \& Shalchi, A. 2014b, Applied Physics Research, 6, 1

Qin, G., \& Wang, Y. 2015, The Astrophysical Journal, 809, 177

Qin, G., Wang, Y., Zhang, M., \& Dalla, S. 2013, Astrophysical Journal, 766, 38

Qin, G., Zhang, M., \& Dwyer, J. R. 2006, Journal of Geophysical Research, 111, 8101

Reinard, A. A., \& Andrews, M. A. 2006, Advances in Space Research, 38, 480

Rice, W. K. M., Zank, G. P., \& Li, G. 2003, Journal of Geophysical Research Atmospheres, 108, 1369

Rouillard, A. P., OdstřCil, D., Sheeley, N. R., et al. 2011, Astrophysical Journal, 735, 660

Rouillard, A. P., Sheeley, N. R., Tylka, A., et al. 2012, Astrophysical Journal, 752, 1750

Schlickeiser, R. 2002, Cosmic ray astrophysics (Springer)

Shalchi, A., Bieber, J. W., Matthaeus, W. H., \& Qin, G. 2004, The Astrophysical Journal, 616, 617

Shalchi, A., Li, G., \& Zank, G. P. 2010, apss, 325, 99

Skilling, J. 1971, The Astrophysical Journal, 170, 265

Temmer, M., Vršnak, B., Rollett, T., et al. 2012, ApJ, 749, 57

Teufel, A., \& Schlickeiser, R. 2003, Astronomy \& Astrophysics, 397, 15

Verkhoglyadova, O. P., Li, G., Zank, G. P., Hu, Q., \& Mewaldt, R. A. 2009, The Astrophysical Journal, 693, 894

Verkhoglyadova, O. P., Li, G., Zank, G. P., et al. 2010, Journal of Geophysical Research, 115, A12103

von Rosenvinge, T. T., Barbier, L. M., Karsch, J., et al. 1995, Space Science Reviews, 71, 155

Wang, Y., \& Qin, G. 2015, The Astrophysical Journal, 806, 252 
Wang, Y., Qin, G., \& Zhang, M. 2012, The Astrophysical Journal, 752, 37

Zank, G. P., Rice, W. K. M., \& Wu, C. C. 2000, Journal of Geophysical Research, 105, 25079

Zhang, M. 1999, The Astrophysical Journal, 513, 409

Zhang, M., Qin, G., \& Rassoul, H. 2009, The Astrophysical Journal, 692, 109 